Ann. Zootech., I972, 21 (I), IO7-III.

NOTE

\title{
OBSERVATIONS PRÉLIMINAIRES SUR LA COMPARAISON DU POTENTIEL DIGESTIF ET DE L'APPÉTIT DES CAPRINS ET DES OVINS EN ZONE TROPICALE ET EN ZONE TEMPÉRÉE
}

\author{
M. CHENOST \\ avec la collaboration technigue de P. Despors \\ Station de Recherches zootechniques, \\ Centre de Recherches agronomiques des Antilles et de la Guyane, I. N. R. A., \\ Petit-Bourg, Guadoloupe
}

Il est généralement admis (I'orbes et al., I937; İrench, 1956; Swift et Bratzler., I959; Alexander et al, rg6z) que les coefficients d'utilisation digestive des aliments est la même pour les différentes espèces de ruminants domestiques ou, tout au plus, que les écarts observés entre espèces sont inférieurs aux écarts dus, pour une espèce donnée, aux erreurs expérimentales.

Jans le cadre plus général d'un travail que nous avons entrepris sur la comparaison de l'appétit et de l'utilisation digestive et métabolique entre ovins, caprins et bovins, nous avons mesuré la digestibilité et les quantités de matière sèche ingérées de fourrages verts et de fourrages conservés en zone tempérée et en zone tropicale humide avec des bóliers et des boucs.

\section{MATÉRIEL ET MÉTHODES}

Deux groupes de comparaison ont été effectués :

Io Mesure de la digestibilité et des quantités ingérées par des moutons et des boucs, au C. R. A. A.G. (Guadeloupe), de 3 repousses de Pangola (Digitaria decumbens) âgées de 30 et 50 jours distribuées en vert à l'auge.

$2^{\circ}$ Mesure de la digestibilité et des quantités ingérées par des moutons, en France et en Guadeloupe et par des boucs, en Guadeloupe, de 4 fourrages de luzerne et de fétuque élevée récoltés au C. R. Z. V. près de Clermont-Ferrand (France), déshydratés puis, compactés, condensés ou comprimés (tabl. I). Les fourrages compactés sont agglomérés par passage sans broyage préalable dans une presse munie d'une filière; les fourrages comprimés sont agglomérés sans broyage dans une presse à piston ; en fin, les fourrages condensés sont agglomérés par passage après broyage dans une presse munie d'une filière. Ces fourrages ainsi préparés ont été envoyés en Guadeloupe par bateau dans des sacs de plastique. 


\section{Animaux.}

Nous avons utilisé des boucs de race Alpine (Chamoisée et Saanen) issus du troupeau expérimental de la Station de Recherches zootechniques du C. R. A. A. G. (Guadeloupe), âgées de I8 à 24 mois, et pesant $45 \mathrm{~kg}$ de moyenne. Les moutons utilisés au C. R. A. A. G., d'origine locale et de race difficile à définir, étaient âgés de i 8 mois et pesaient en moyenne $35 \mathrm{~kg}$. Les moutons utilisés au C. R. Z.V. près de Clermont-Ferrand (France métropolitaine), de race Texel, étaient âgés de 4 ans et pesaient $80 \mathrm{~kg}$ en moyenne.

\section{Dispositif expérimental et mesures.}

Chaque lot était constitué de 4 animaux maintenus en cage à digestibilité et de 2 animaux en case au sol. Toutefois, le lot de moutons utilisé en Guadeloupe n'était constitué que de 5 animaux.

La conduite des essais de digestibilité a étéla même que celle utilisée par Demarquilly et WEISs (I970) au C. R. Z.V. Chaque période de mesure effectuée sur fourrage vert dure une semaine ( 6 jours de collecte) et est précédée d'une période préexpérimentale d'une semaine, celle effectuée sur fourrage conservé dure ro jours et est précédée d'une période préexpérimentale de ro jours.

Les fourrages sont distribués à volonté (Io p. Ioo de refus) en deux repas, l'un à 9 heures I'autre à 16 heures.

Nous avons calculé les coefficients d'utilisation digestive et les quantités ingérées correspondant à chaque période de mesure. Les échantillons représentatifs du fourrage offert, du fourrage refusé et des fèces correspondant à chaque période de mesure ont été analysés pour déterminer les teneurs en cendres, en matières azotées totales et en cellulose brute Weende.

\section{RÉSULTATS ET DISCUSSION}

\section{Coefficients d'utilisation digestive}

La digestibilité de la matière organique des fourrages a été en moyenne la même chez le bouc et chez le mouton, les seules différences significatives ont été observées avec deux repousses de Pangola en faveur des moutons (tabl. r). Elle a également été la même avec les moutons du C. R. A. A. G. et avec ceux du C. R. Z. V. La variabilité des mesures n'est pas différente suivant les espèces ; toutefois, au C. R. A. A. G., 3 mesures obtenues sur moutons et 2 mesures obtenues sur boucs ont été entachées de coefficients de variabilité élevés (tabl. I).

La digestibilité de la cellulose brute a été, en Guadeloupe, en moyenne la même chez le bouc et chez le mouton; cependant, des différences significatives ont été observées en faveur des moutons avec deux repousses de pangola et en faveur des boucs avec la luzerne condensée. La digestibilité des matières azotées a été un peu plus élevée en moyenne chez le mouton que chez le bouc mais la différence n'est pas significative.

Ces résultats, confirmant ceux de Forbes et al. (1937), Swift et Braztler (i959) et AlexanDER et al. (1962), montrent que, tout au moins pour des fourrages de faible digestibilité tels que ceux que nous avons étudiés, le potentiel digestif des boucs et des moutons et très voisin et que les différences ducs à l'espèce animale sont inférieures à celles dues aux erreurs expérimentales ou aux différences individuelles à l'intérieur de la même espèce.

Nous avons observé des différences importantes dans les digestibilités de la cellulose brute et des matières azotées des fourrages conservés entre les moutons du C. R. Z.V. et les moutons du C. R. A. A. G. (tabl. I). La digestibilité de la cellulose brute de la fétuque élevée comprimée et de la luzerne condensée a été plus élevée au C. R. A. A. G. qu'au C. R. Z. V. et la digestibilité des matières azotées de la fétuque élevée comprimée et des deux luzernes a été plus faible au C. R. A. A. G. qu'au C. R. Z. V.

Ces différences ne semblent pas dues à des erreurs d'analyse ou d'échantillonnage et il serait 


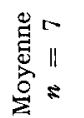

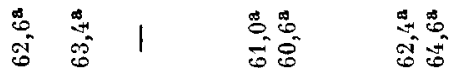

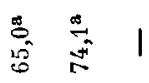

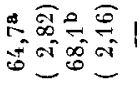

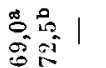

$\mathbb{s}_{-\infty}^{\infty}=$

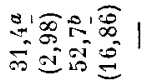

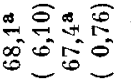

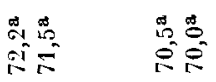

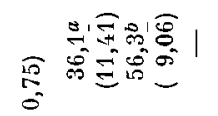

$\Xi$

官<smiles>[CH]1CCCC1</smiles><smiles>[CH]1[CH]C[CH]1</smiles>

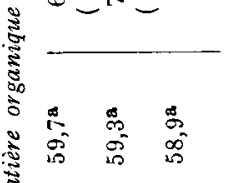

i

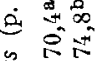

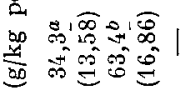

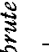
农

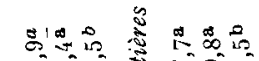

in

in

5

8

8

政

\& 5 :

$\sin 2$

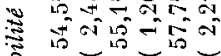

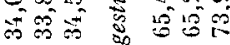

要

盾

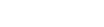
a<smiles>[Li][CaH]</smiles>

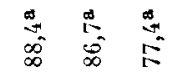

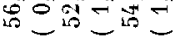

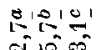

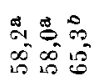

㐔

ㅂ.

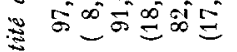

총응

कू

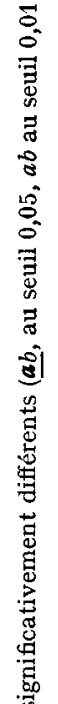

․ำ

$\ddot{a}: \vec{a}$

| | |

as or

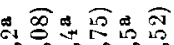

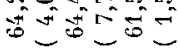

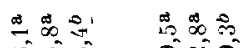

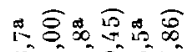

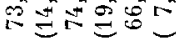

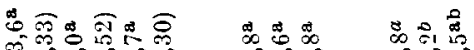

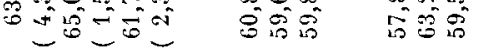

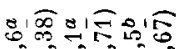

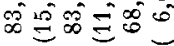

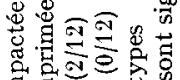

\section{.}

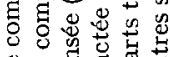

语

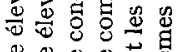

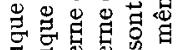

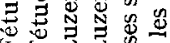

-

$+$

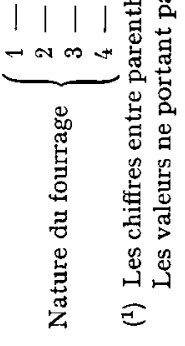


intéressant de poursuivre l'étude comparative de manière à connaitre la cause de ces différences. Il est en effet possible que les clifférences clans les quantités ingérées aient été en partie responsables des différences dans les coefficients de cligestibilité.

\section{Quantités de matière sèche ingérées}

Les moutons ont ingéré en quantité significativement plus élevée $(\mathrm{P}<0,05)$ que les boucs les 3 fourrages verts de Pangola du C. R. A. A. G. : en moyenne 57,5 contre $32,9 \mathrm{~g} / \mathrm{kg} \mathrm{P}^{0,75}$ (tabl. I).

Les moutons du C. R. Z. V. ont ingéré 2 des + fourrages déshydratés en quantité significativement plus faible $\left(\mathrm{P}^{P}<\mathrm{O}\right.$,or $)$ que les moutons et les boucs du C. R. A. A. G., les quantités ingérées par ces deux catégories d'animaux n'étant pas significativement différentes : respectivement en moyenne 88,4 et $86,7 \mathrm{~g} / \mathrm{kg} \mathrm{P}^{0,75}$.

Les différences de quantités ingérées par les moutons créoles utilisés au C. R. A. A. G. et les moutons de race Texel utilisés au C. R.Z.V. doivent résulter, d'une part, de différences de format, les moutons créoles étant beaucoup plus légers, d'autre part, des différences d'état d'engraissement, les moutons utilisés au C. R. Z.V. recevant des fourrages de qualité très souvent supérieure à ceux distribués aux moutons créoles.

Le comportement différent entre boucs et moutons créoles est plus difficile à expliquer. Nous nous proposons d'en analyser les causes, d'autant plus que nous avons utilisé systématiquement les boucs issus de notre troupeau laitier pour mesurer la valeur alimentaire (digestibilité - quantités ingérées) des fourrages locaux.

La variabilité des quantités ingérées a été plus élevée en moyenne chez les boucs que chez les moutons tant pour les fourrages verts que pour les fourrages conservés, elle a été plus faible chez les moutons du Centre de Clermont-Ferrand que chez les moutons et les boucs du Centre des Antilles.

Reçu pour publication en juillet 1971.

\section{SUMMARY}

\section{COMPARISON OF THE DIGESTIVE ABILTTY AND THE, APPETITE OF GOATS AND SHEEP IN TROPICAL AND IN TEMPERATE ZONES. PRELIMINARY STUDY}

The digestibilities and dry matter intake of two groups of forages were compared (table I ):

- forages harvested and dehydrated in France;

- green forages harvested in Guadaloupe (Digitaria decumbens).

The measurements wore made.

- in France, using sheep of the Alpine brecd (dehydrated forages) forages).

- in Guadaloupe, using creole sheep and goats of the Texel breed (dehydrated and green

There was no significant difference between the animal species and the geographical sites as regards the digestibility coefficients of the organic matter. The digestibility coefficients of crude fiber and crude protein measured in Guadaloupe were similar for goats and sheep. On the other hand, the digestibility coefficients of crude fiber were higher and those of crude protein lower in Guadaloupe than in France (table $\mathrm{I}$ ).

The variation of the dry matter intake was more important in Guadaloupe than in France. When using dehydrated forages, the dry matter intake was higher in sheep than in goats (table I). 


\section{RÉFÉRENCES BIBI,IOGRAPHIQUES}

Alexander R. A., Hentges J. F., Mc Call J. T., Ash W. O., I962. Comparative digestibility of nutrients in roughages by cattle and sheep. J. Anim. Sci., 21, 373.

Demarquilly C., Weiss PH., I970. Tableaux de la valeur alimentaire des fourrages. I. N. R. A. Étude S. E. I., $\mathrm{n}^{\circ} 42$.

FRENCH M. H., 1956. The effect of restricted intake on the digestibility of hays by East African hair sheep and zebu oxen. Empire J. Exp. Agr., 24, 235.

Swift R. W., Bratzler J. W., I959. A comparison of the digestibility of forages by cattle and by sheep. Pa. A gric. Exp. Sta. Bull., 651. 